\title{
Coded equivalence of one-sided topological Markov shifts
}

\author{
Kengo Matsumoto \\ Department of Mathematics \\ Joetsu University of Education \\ Joetsu, 943-8512, Japan
}

November 30, 2020

\begin{abstract}
We introduce a notion of coded equivalence in one-sided topological Markov shifts. The notion is inspired by coding theory. One-sided topological conjugacy implies coded equivalence. We will show that coded equivalence implies continuous orbit equivalence of one-sided topological Markov shifts.
\end{abstract}

2020Mathematics Subject Classification: Primary 94A45, 68Q45; Secondary 37B10, 68R15, 37A55.

Keywords and phrases: code, prefix code, coded equivalence, topological Markov shift, orbit equivalence.

In [13], the author introduced a notion of continuous orbit equivalence of one-sided topological Markov shifts. The definition of the equivalence relation was primary inspired by orbit equivalence theory of minimal homeomorphisms on Cantor sets established by Giordano-Putnam-Skau [9] (cf. [10], etc.). Through the studies of classifications of continuous orbit equivalence of one-sided topological Markov shifts, several interesting relationships with other areas of mathematics, $C^{*}$-algebras, groupoids, infinite discrete groups, etc. have been clarified (cf. [15], [16], etc. ). As a result, H. Matui and the author have succeeded to classify irreducible one-sided topological Markov shifts under continuous orbit equivalence ([15]). However, there is no known any systematic method to give rise to continuous orbit equivalence of one-sided topological Markov shifts. In this paper, we introduce a notion of coded equivalence in one-sided topological Markov shifts. The definition of the coded equivalence is inspired by coding theory of formal language theory. Although relationship between symbolic dynamics and coding theory has been studied by many authors, for example [1], [3], [4, 6], 7], 8], etc, the coded equivalence treated in this paper has not seen in any other papers than this.

We will study the coded equivalence from the view point of symbolic dynamical systems. It is well-known that topological conjugacy of symbolic dynamical systems is given by a sliding block code (cf. [11, [12]). We will also introduce a notion of moving block code, that is a generalization of sliding block code. We will then see that one-sided topological conjugacy implies coded equivalence. As a main result of the paper, we will show that the coded equivalence implies continuous orbit equivalence of one-sided topological Markov shifts (Theorem 14). We therefore know a close relationship between coding theory 
and continuous orbit equivalence theory of one-sided topological Markov shifts. Several examples of coded equivalent topological Markov shifts will be presented.

We will first provide several terminology and notation. Let us denote by $\mathbb{N}$ and $\mathbb{Z}_{+}$ the set of positive integers and the set of nonnegative integers, respectively. Let $A=$ $[A(i, j)]_{i, j=1}^{N}$ be an irreducible non permutation matrix over $\{0,1\}$. Let us denote by $\Sigma_{A}$ the set $\{1,2, \ldots, N\}$. Let $X_{A}$ be the set of right one-sided infinite sequences $\left(x_{n}\right)_{n \in \mathbb{N}}$ of $\Sigma_{A}$ such that $A\left(x_{n}, x_{n+1}\right)=1$ for all $n \in \mathbb{N}$. The set $X_{A}$ is endowed with its product topology so that it is a homeomorphic to a Cantor discontinuum. It has a natural shift operation $\sigma_{A}$ defined by $\sigma_{A}\left(\left(x_{n}\right)_{n \in \mathbb{N}}\right)=\left(x_{n+1}\right)_{n \in \mathbb{N}}$. The topological dynamical system $\left(X_{A}, \sigma_{A}\right)$ is called the one-sided topological Markov shift defined by the matrix $A$. The space $X_{A}$ is called the shift space of $\left(X_{A}, \sigma_{A}\right)$. Let us denote by $B_{k}\left(X_{A}\right)$ the set of admissible words of $X_{A}$ with length $k$. We put $B_{*}\left(X_{A}\right)=\cup_{k=0}^{\infty} B_{k}\left(X_{A}\right)$, where $B_{0}\left(X_{A}\right)$ denotes the empty word. For a word $\mu=\left(\mu_{1}, \ldots, \mu_{m}\right) \in B_{m}\left(X_{A}\right)$, let us denote by $U_{\mu}$ the cylinder set

$$
U_{\mu}=\left\{\left(x_{n}\right)_{n \in \mathbb{N}} \in X_{A} \mid x_{1}=\mu_{1}, \ldots, x_{m}=\mu_{m}\right\} .
$$

A code $\mathcal{C}$ of $X_{A}$ is a nonempty subset $\mathcal{C} \subset B_{*}\left(X_{A}\right)$ such that for any equality

$$
\omega\left(i_{1}\right) \omega\left(i_{2}\right) \cdots \omega\left(i_{k}\right)=\omega\left(j_{1}\right) \omega\left(j_{2}\right) \cdots \omega\left(j_{n}\right)
$$

of words with $\omega\left(i_{1}\right), \omega\left(i_{2}\right), \cdots, \omega\left(i_{k}\right), \omega\left(j_{1}\right), \omega\left(j_{2}\right), \cdots, \omega\left(j_{n}\right) \in \mathcal{C}$, one has

$$
n=k \quad \text { and } \quad \omega\left(i_{m}\right)=\omega\left(j_{m}\right), \quad m=1,2, \ldots, n .
$$

A prefix code is a code such that no word in it can be the beginning of another ( $\mathrm{cf}$. [2], 3]). For example, let $A=\left[\begin{array}{ll}1 & 1 \\ 1 & 1\end{array}\right]$ and $\Sigma_{A}=\{1,2\}$. Consider $\mathcal{C}=\{1,21,22\}$ and $\mathcal{C}^{\prime}=\{1,12,22\}$. We see that $\mathcal{C}$ is a prefix code, whereas $\mathcal{C}^{\prime}$ is a code but not a prefix code. The following lemma is easy to prove.

Lemma 1. Let $\mathcal{C}=\{\omega(1), \omega(2), \ldots, \omega(M)\} \subset B_{*}\left(X_{A}\right)$ be a finite set of admissible words of $X_{A}$. Then $\mathcal{C}$ is a prefix code if and only if $U_{\omega(i)} \cap U_{\omega(j)}=\emptyset$ for $i \neq j$.

For a prefix code $\mathcal{C}=\{\omega(1), \omega(2), \ldots, \omega(M)\} \subset B_{*}\left(X_{A}\right)$, we denote by $\Sigma_{A(\mathcal{C})}$ the set $\{1,2, \ldots, M\}$. Let us denote by $\ell(i)$ the length of the word $\omega(i), i \in \Sigma_{A(\mathcal{C})}$. The word $\omega(i)$ is written

$$
\omega(i)=\omega_{1}(i) \omega_{2}(i) \cdots \omega_{\ell(i)}(i) \in B_{\ell(i)}\left(X_{A}\right) \quad \text { for some } \quad \omega_{j}(i) \in \Sigma_{A}
$$

For the word $\omega(i)$, define $\sigma_{A}(\omega(i)) \in B_{\ell(i)-1}\left(X_{A}\right)$ by setting

$$
\sigma_{A}(\omega(i))=\omega_{2}(i) \omega_{3}(i) \cdots \omega_{\ell(i)}(i)
$$

We will introduce a notion of right Markov code in the following way.

Definition 2. Let $\mathcal{C}=\{\omega(1), \omega(2), \ldots, \omega(M)\} \subset B_{*}\left(X_{A}\right)$ be a prefix code. The prefix code $\mathcal{C}$ is called a right Markov code for $\left(X_{A}, \sigma_{A}\right)$ if it satisfies the following three conditions: 
(i) (unique factorization) For any $\gamma \in B_{*}\left(X_{A}\right)$, there exists a word $\eta \in B_{*}\left(X_{A}\right)$ such that $\gamma \eta \in B_{*}\left(X_{A}\right)$, and there exists a unique finite sequence $\left(i_{1}, i_{2}, \ldots, i_{k}\right) \in\left(\Sigma_{A(\mathcal{C})}\right)^{k}$ such that

$$
\gamma \eta=\omega\left(i_{1}\right) \omega\left(i_{2}\right) \cdots \omega\left(i_{k}\right)
$$

(ii) (shift invariance) There exists $L \in \mathbb{N}$ such that for any $i_{1}, i_{2}, \ldots, i_{L} \in \Sigma_{A(\mathcal{C})}$ with $\omega\left(i_{1}\right) \omega\left(i_{2}\right) \cdots \omega\left(i_{L}\right) \in B_{*}\left(X_{A}\right)$, there exists $j_{1}, j_{2}, \ldots, j_{k} \in \Sigma_{A(\mathcal{C})}$ such that

$$
\sigma_{A}\left(\omega\left(i_{1}\right)\right) \omega\left(i_{2}\right) \cdots \omega\left(i_{L}\right)=\omega\left(j_{1}\right) \omega\left(j_{2}\right) \cdots \omega\left(j_{k}\right),
$$

where $k$ depends on the finite sequence $\left(i_{1}, i_{2}, \ldots, i_{L}\right)$.

(iii) (irreducibility) For any ordered pair $\omega(i), \omega(j) \in \mathcal{C}$, there exist $n_{1}, n_{2} \ldots, n_{l} \in \Sigma_{A(\mathcal{C})}$ such that

$$
\omega(i) \omega\left(n_{1}\right) \omega\left(n_{2}\right) \cdots \omega\left(n_{l}\right) \omega(j) \in B_{*}\left(X_{A}\right)
$$

We call (i), (ii) and (iii) unique factorization property, shift equivalence property and irreducible condition, respectively.

Remark 3. In the definition of right Markov code, we do not necessarily assume that $\omega(i) \in \mathcal{C}$ implies $\sigma(\omega(i)) \in \mathcal{C}$. Hence in the above definition (ii), the word $\sigma_{A}(\omega(i))$ does not necessarily belong to the set $\mathcal{C}$ when $\omega(i) \in \mathcal{C}$.

Lemma 4. Let $\mathcal{C}=\{\omega(1), \omega(2), \ldots, \omega(M)\} \subset B_{*}\left(X_{A}\right)$ be a prefix code. The following assertions are equivalent.

(i) $\mathcal{C}$ satisfies the unique factorization property (i) of Definition 2 .

(ii) For any $x=\left(x_{n}\right)_{n \in \mathbb{N}} \in X_{A}$ there exists a unique increasing sequence $1<k_{1}<k_{2}<$ $\cdots$ of positive integers and $i_{1}, i_{2}, \cdots \in \Sigma_{A(\mathcal{C})}$ such that

$$
x_{\left[1, k_{1}\right)}=\omega\left(i_{1}\right), \quad x_{\left[k_{1}, k_{2}\right)}=\omega\left(i_{2}\right), \quad \ldots, \quad x_{\left[k_{n}, k_{n+1}\right)}=\omega\left(i_{n+1}\right), \quad \ldots
$$

That is, $x$ has a unique factorization:

$$
x=\omega\left(i_{1}\right) \omega\left(i_{2}\right) \cdots \omega\left(i_{n}\right) \cdots
$$

Proof. (i) $\Longrightarrow$ (ii): Assume that $\mathcal{C}=\{\omega(1), \omega(2), \ldots, \omega(M)\} \subset B_{*}\left(X_{A}\right)$ satisfies the unique factorization property (i) of Definition 2. Put $K_{0}=\max \left\{\ell(i) \mid i \in \Sigma_{A(\mathcal{C})}\right\}$, where $\ell(i)$ denotes the length of $\omega(i)$. Take an increasing sequence $1<m_{1}<m_{2}<\cdots$ of positive integers such that $n \cdot K_{0}<m_{n}$ for $n \in \mathbb{N}$. Take an arbitrary $x=\left(x_{n}\right)_{n \in \mathbb{N}} \in X_{A}$. For the word $x_{\left[1, m_{n}\right]} \in B_{m_{n}}\left(X_{A}\right)$ for each $n \in \mathbb{N}$, by the unique factorization property (i) of Definition 2, there exists $\eta(n) \in B_{*}\left(X_{A}\right)$ such that $x_{\left[1, m_{n}\right]} \eta(n) \in B_{*}\left(X_{A}\right)$, and there exists a unique finite sequence $\left(i_{1}(n), i_{2}(n), \ldots, i_{k_{n}}(n)\right) \in\left(\Sigma_{A(\mathcal{C})}\right)^{k_{n}}$ such that

$$
x_{\left[1, m_{n}\right]} \eta(n)=\omega\left(i_{1}(n)\right) \omega\left(i_{2}(n)\right) \cdots \omega\left(i_{k_{n}}(n)\right) .
$$

Since $\mathcal{C}$ is a prefix code, we see that

$$
\begin{aligned}
\omega\left(i_{1}(1)\right) & =\omega\left(i_{1}(n)\right) & & \text { for all } n \geq 1, \\
\omega\left(i_{2}(2)\right) & =\omega\left(i_{2}(n)\right) & & \text { for all } n \geq 2, \\
\cdots & =\cdots & & \\
\omega\left(i_{\ell}(\ell)\right) & =\omega\left(i_{\ell}(n)\right) & & \text { for all } n \geq \ell .
\end{aligned}
$$


Take $\xi(n) \in X_{A}$ such that $x_{\left[1, m_{n}\right]} \eta(n) \xi(n) \in X_{A}$. Put $x(n)=x_{\left[1, m_{n}\right]} \eta(n) \xi(n) \in X_{A}$ for $n \in \mathbb{N}$. Then $x(n)$ converges to $x$ and we have

$$
x=\omega\left(i_{1}(1)\right) \omega\left(i_{2}(2)\right) \cdots \omega\left(i_{\ell}(\ell)\right) \cdots
$$

The factorization is unique, because $\mathcal{C}$ is a prefix code.

(ii) $\Longrightarrow($ i): The assertion is obvious.

For an $N \times N$ irreducible matrix $A=[A(i, j)]_{i, j=1}^{N}$ with entries in $\{0,1\}$, an associated directed graph $G_{A}=\left(V_{A}, E_{A}\right)$ is defined in the following way. The vertex set $V_{A}=\Sigma_{A}(=$ $\{1,2, \ldots, N\})$. If $A(i, j)=1$, then a directed edge from the vertex $i$ to the vertex $j$ is defined. The edge set $E_{A}$ consists of such edges. The transition matrix of the graph $G_{A}$ is the original matrix $A$. Hence the shift space $X_{A}$ consists of right infinite sequences of concatenating vertices in the graph $G_{A}$.

\section{Example 5.}

0. Let $A=[A(i, j)]_{i, j=1}^{N}$ be an $N \times N$ irreducible matrix with entries in $\{0,1\}$. Define $\mathcal{C}_{0}=\Sigma_{A}$ the set of admissible words of length 1 . Then $\mathcal{C}_{0}$ is a right Markov code. It is called the trivial right Markov code for $\left(X_{A}, \sigma_{A}\right)$.

1. Let $A_{1}=\left[\begin{array}{ll}1 & 1 \\ 1 & 0\end{array}\right]$. Define $\mathcal{C}_{1}=\{1,21\}$. Then $\mathcal{C}_{1}$ is a right Markov code. The directed graph $G_{A_{1}}$ is Figure 1 .

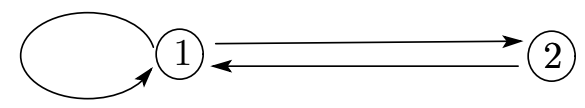

Figure 1: The directed graph $G_{A_{1}}$

2. Let $A_{2}=\left[\begin{array}{lll}0 & 1 & 0 \\ 0 & 0 & 1 \\ 1 & 1 & 0\end{array}\right]$. Define $\mathcal{C}_{2}=\{12,23,323,312\}$. Then $\mathcal{C}_{2}$ is a right Markov code.

3. Let $A_{3}=\left[\begin{array}{lll}1 & 1 & 1 \\ 1 & 0 & 1 \\ 1 & 0 & 0\end{array}\right]$. Define $\mathcal{C}_{3}=\{1,21,31,231\}$. Then $\mathcal{C}_{3}$ is a right Markov code.

The directed graphs $G_{A_{2}}$ and $G_{A_{3}}$ are Figure 2 .

The following lemma shows that a one-sided full shift has only trivial right Markov code.

Lemma 6. Let $\mathcal{C} \subset B_{*}\left(X_{A}\right)$ be a right Markov code for $\left(X_{A}, \sigma_{A}\right)$. Suppose that $X_{A}$ has a fixed point $a^{\infty}=(a, a, a, \ldots) \in X_{A}$. We then have $a^{m}=\overbrace{a \cdots a}^{m} \in \mathcal{C}$ if and only if $m=1$.

Proof. Let $\mathcal{C}=\{\omega(1), \omega(2), \ldots, \omega(M)\} \subset B_{*}\left(X_{A}\right)$ be a right Markov code for $\left(X_{A}, \sigma_{A}\right)$. By Lemma 4, there uniquely exists $i_{1}, i_{2}, \cdots \in \Sigma_{A(\mathcal{C})}$ such that the fixed point $a^{\infty}$ is uniquely factorized as

$$
a^{\infty}=\omega\left(i_{1}\right) \omega\left(i_{2}\right) \cdots \omega\left(i_{n}\right) \cdots
$$



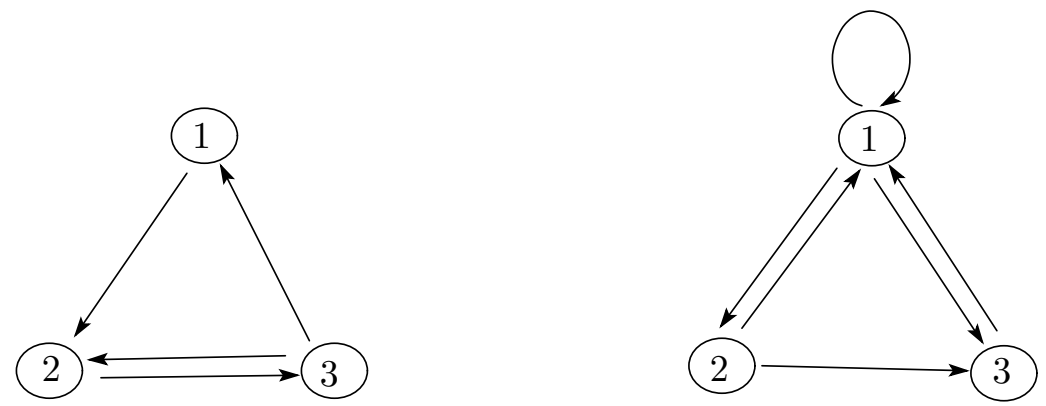

Figure 2: The directed graphs $G_{A_{2}}$ and $G_{A_{3}}$

Suppose that

$$
\omega\left(i_{1}\right)=\overbrace{a \cdots a}^{m_{1}}, \quad \omega\left(i_{2}\right)=\overbrace{a \cdots a}^{m_{2}}, \quad \ldots
$$

Since the code $\mathcal{C}$ is a prefix code, we know that $m_{1}=m_{2}=\cdots$ putting $m_{0}$. Suppose that $m_{0}>1$. By the shift invariance property of right Markov code, there exists $L \in \mathbb{N}$ such that there exist $j_{1}, j_{2}, \ldots, j_{k} \in \Sigma_{A(\mathcal{C})}$ satisfying

$$
\sigma_{A}\left(a^{m_{0}}\right) \overbrace{a^{m_{0}} \cdots a^{m_{0}}}^{L-1}=\omega\left(j_{1}\right) \omega\left(j_{2}\right) \cdots \omega\left(j_{k}\right) .
$$

The words $\omega\left(j_{1}\right), \omega\left(j_{2}\right), \ldots, \omega\left(j_{k}\right)$ appeared in the right hand side of (2) must be $a^{m_{0}}$, because $\mathcal{C}$ is a prefix code. Hence we see that

$$
a^{m_{0}-1} \overbrace{a^{m_{0}} \cdots a^{m_{0}}}^{L-1}=\overbrace{a^{m_{0}} a^{m_{0}} \cdots a^{m_{0}}}^{k}
$$

so that $m_{0}-1+(L-1) m_{0}=k \cdot m_{0}$, proving $m_{0}=1$. This implies that $a^{m}=\overbrace{a \cdots a}^{m} \in \mathcal{C}$ if and only if $m=1$.

Let us denote by $\left(X_{[N]}, \sigma_{[N]}\right)$ the full $N$-shift over $\Sigma_{[N]}=\{1,2, \ldots, N\}$

Proposition 7. A one-sided full shift has only trivial right Markov code.

Proof. Let $\mathcal{C}$ be a right Markov code for $\left(X_{[N]}, \sigma_{[N]}\right)$. By Lemma 6 , we see that $\Sigma_{[N]} \subset \mathcal{C}$. As $\mathcal{C}$ is a prefix code, there are no other words in $\mathcal{C}$ than $\Sigma_{[N]}$.

Let $\mathcal{C}=\{\omega(1), \omega(2), \ldots, \omega(M)\} \subset B_{*}\left(X_{A}\right)$ be a right Markov code for $\left(X_{A}, \sigma_{A}\right)$. We write $\omega(i)=\left(\omega_{1}(i), \omega_{2}(i), \ldots, \omega_{\ell(i)}(i)\right) \in B_{\ell(i)}\left(X_{A}\right)$ with $\omega_{j}(i) \in \Sigma_{A}$. We put

$$
s(\omega(i))=\omega_{1}(i) \in \Sigma_{A}, \quad r(\omega(i))=\omega_{\ell(i)}(i) \in \Sigma_{A} .
$$

Define an $M \times M$ matrix $A(\mathcal{C})=[A(\mathcal{C})(i, j)]_{i, j=1}^{M}$ with entries in $\{0,1\}$ by setting

$$
A(\mathcal{C})(i, j)=A(r(\omega(i)), s(\omega(j))), \quad i, j \in \Sigma_{A(\mathcal{C})}
$$


This means that

$$
A(\mathcal{C})(i, j)= \begin{cases}1 & \text { if } \omega(i) \omega(j) \in B_{*}\left(X_{A}\right), \\ 0 & \text { otherwise. }\end{cases}
$$

Hence we have a one-sided topological Markov shift $\left(X_{A(\mathcal{C})}, \sigma_{A(\mathcal{C})}\right)$ from a right Markov code $\mathcal{C}$ for $\left(X_{A}, \sigma_{A}\right)$.

Lemma 8. Suppose that $A$ is irreducible and not any permutation. Then the matrix $A(\mathcal{C})$ is irreducible and not any permutation.

Proof. By the irreducible condition (iii) of Definition 2, it is direct to see that the matrix $A(\mathcal{C})$ is irreducible. It suffices to show that $A(\mathcal{C})$ is not any permutation. As $A$ is irreducible and not any permutation, for any $x_{1} \in \Sigma_{A}$, there exists a finite sequence $x_{2}, \ldots, x_{k}, x_{k+1}, x_{k+1}^{\prime} \in \Sigma_{A}$ with $x_{k+1} \neq x_{k+1}^{\prime}$ such that $\left(x_{1}, x_{2}, \ldots, x_{k}, x_{k+1}\right) \in B_{k+1}\left(X_{A}\right)$ and $\left(x_{1}, x_{2}, \ldots, x_{k}, x_{k+1}^{\prime}\right) \in B_{k+1}\left(X_{A}\right)$. Extend the admissible words $\left(x_{1}, x_{2}, \ldots, x_{k}, x_{k+1}\right)$ and $\left(x_{1}, x_{2}, \ldots, x_{k}, x_{k+1}^{\prime}\right)$ to its right infinitely as elements of $X_{A}$. We denote them by $x, x^{\prime} \in X_{A}$, respectively, so that $x \neq x^{\prime}$. By Lemma 4 there exist $n \in \mathbb{N}$ and

$$
\omega\left(i_{1}\right) \omega\left(i_{2}\right) \cdots \omega\left(i_{n-1}\right) \omega\left(i_{n}\right), \quad \omega\left(i_{1}\right) \omega\left(i_{2}\right) \cdots \omega\left(i_{n-1}\right) \omega\left(i_{n}^{\prime}\right) \in B_{*}\left(X_{A}\right)
$$

for $x, x^{\prime}$ so that $\left(i_{1}, i_{2}, \ldots, i_{n-1}, i_{n}\right),\left(i_{1}, i_{2}, \ldots, i_{n-1}, i_{n}^{\prime}\right) \in B_{n}(A(\mathcal{C}))$, such that $i_{n} \neq i_{n}^{\prime}$. As $A(\mathcal{C})$ is irreducible, any $i_{k} \in \Sigma_{A(\mathcal{C})}$ goes to $i_{1}$, so that $i_{k}$ has distinct followers in $X_{A(\mathcal{C})}$. This means that the matrix $A(\mathcal{C})$ is not any permutation.

Two one-sided topological Markov shifts $\left(X_{A}, \sigma_{A}\right)$ and $\left(X_{B}, \sigma_{B}\right)$ are said to be topologically conjugate if there exists a homeomorphism $h: X_{A} \longrightarrow X_{B}$ such that $h \circ \sigma_{A}=\sigma_{B} \circ h$. The homeomorphism $h: X_{A} \longrightarrow X_{B}$ is called a topological conjugacy. Now we will introduce a notion of coded equivalence in one-sided topological Markov shifts.

Definition 9. Let $A, B$ be irreducible non permutation matrices. The one-sided topological Markov shifts $\left(X_{A}, \sigma_{A}\right)$ and $\left(X_{B}, \sigma_{B}\right)$ are said to be elementary coded equivalent if there exist a right Markov code $\mathcal{C}_{1}$ for $\left(X_{A}, \sigma_{A}\right)$ and a right Markov code $\mathcal{C}_{2}$ for $\left(X_{B}, \sigma_{B}\right)$ such that the one-sided topological Markov shifts $\left(X_{A\left(\mathcal{C}_{1}\right)}, \sigma_{A\left(\mathcal{C}_{1}\right)}\right)$ and $\left(X_{B\left(\mathcal{C}_{2}\right)}, \sigma_{B\left(\mathcal{C}_{2}\right)}\right)$ are topologically conjugate. It is written $\left(X_{A}, \sigma_{A}\right) \underset{\text { code }}{\approx}\left(X_{B}, \sigma_{B}\right)$. If there exists a finite sequence $A_{0}, A_{1}, A_{2}, \ldots A_{m}$ of irreducible non permutation matrices such that $A=A_{0}, A_{m}=B$ and

$$
\left(X_{A_{0}}, \sigma_{A_{0}}\right) \underset{\text { code }}{\approx}\left(X_{A_{1}}, \sigma_{A_{1}}\right) \underset{\text { code }}{\approx} \cdots \underset{\text { code }}{\approx}\left(X_{A_{m-1}}, \sigma_{A_{m-1}}\right) \underset{\text { code }}{\underset{\approx}{\approx}}\left(X_{A_{m}}, \sigma_{A_{m}}\right),
$$

then $\left(X_{A}, \sigma_{A}\right)$ and $\left(X_{B}, \sigma_{B}\right)$ are said to be coded equivalent. It is written $\left(X_{A}, \sigma_{A}\right)$ code $\left(X_{B}, \sigma_{B}\right)$.

Lemma 10. (i) Let $A, B$ be irreducible non permutation matrices. If the one-sided topological Markov shifts $\left(X_{A}, \sigma_{A}\right)$ and $\left(X_{B}, \sigma_{B}\right)$ are topologically conjugate, then they are coded equivalent.

(ii) Let $\mathcal{C} \subset B_{*}\left(X_{A}\right)$ be a right Markov code for $\left(X_{A}, \sigma_{A}\right)$. Then $\left(X_{A}, \sigma_{A}\right)$ and $\left(X_{A(\mathcal{C})}, \sigma_{A(\mathcal{C})}\right)$ are coded equivalent. 
Proof. (i) The assertion is clear by considering trivial right Markov codes for each of $\left(X_{A}, \sigma_{A}\right)$ and $\left(X_{B}, \sigma_{B}\right)$.

(ii) The assertion is also clear by considering trivial right Markov codes for each of $\left(X_{A}, \sigma_{A}\right)$ and $\left(X_{A(\mathcal{C})}, \sigma_{A(\mathcal{C})}\right)$.

We will next introduce a notion of moving block code between one-sided topological Markov shifts. It is a generalization of sliding block codes between one-sided topological Markov shifts and gives rise to a coded equivalence.

Let $\mathcal{C}=\{\omega(1), \omega(2), \ldots, \omega(M)\} \subset B_{*}\left(X_{A}\right)$ be a right Markov code for $\left(X_{A}, \sigma_{A}\right)$. Let us define $\kappa_{\mathcal{C}}: \mathcal{C} \longrightarrow \Sigma_{A(\mathcal{C})}$ by $\kappa_{\mathcal{C}}(\omega(i))=i$ for $i \in \Sigma_{A(\mathcal{C})}$, and a homeomorphism $h_{\mathcal{C}}: X_{A} \longrightarrow X_{A(\mathcal{C})}$ by setting

$$
h_{\mathcal{C}}\left(\omega\left(i_{1}\right) \omega\left(i_{2}\right) \cdots \omega\left(i_{n}\right) \cdots\right)=\left(i_{1}, i_{2}, \ldots, i_{n}, \ldots\right) \quad \text { for } \quad \omega\left(i_{1}\right) \omega\left(i_{2}\right) \cdots \omega\left(i_{n}\right) \cdots \in X_{A} .
$$

We call the homeomorphism $h_{\mathcal{C}}: X_{A} \longrightarrow X_{A(\mathcal{C})}$ the standard coding homeomorphism and write $h_{\mathcal{C}}=\kappa_{\mathcal{C}}^{\infty}$.

Let us denote by $B_{n}(\mathcal{C})$ the set of concatenated $n$ words of the code $\mathcal{C}$, that is

$$
B_{n}(\mathcal{C})=\left\{\omega\left(i_{1}\right) \cdots \omega\left(i_{n}\right) \in B_{*}\left(X_{A}\right) \mid i_{1}, \ldots, i_{n} \in \Sigma_{A(\mathcal{C})}\right\} .
$$

Definition 11. Let $\mathcal{C}_{1}=\left\{\omega(1), \omega(2), \ldots, \omega\left(M_{1}\right)\right\} \subset B_{*}\left(X_{A}\right)$ be a right Markov code for $\left(X_{A}, \sigma_{A}\right)$ and $\mathcal{C}_{2}=\left\{\xi(1), \xi(2), \ldots, \xi\left(M_{2}\right)\right\} \subset B_{*}\left(X_{B}\right)$ be a right Markov code for $\left(X_{B}, \sigma_{B}\right)$. Let $\Phi: B_{n+1}\left(X_{A\left(\mathcal{C}_{1}\right)}\right) \longrightarrow \Sigma_{B\left(\mathcal{C}_{2}\right)}$ be a block map in the ordinary sense (cf. [12]). Then a coded block map $\Phi_{\mathcal{C}}: B_{n+1}\left(\mathcal{C}_{1}\right) \longrightarrow B_{1}\left(\mathcal{C}_{2}\right)\left(=\mathcal{C}_{2}\right)$ is defined by

$$
\Phi_{C}\left(\omega\left(i_{1}\right) \omega\left(i_{2}\right) \ldots \omega\left(i_{n+1}\right)\right)=\kappa_{\mathcal{C}_{2}}^{-1} \circ \Phi\left(i_{1}, i_{2}, \ldots, i_{n+1}\right), \quad i_{1}, i_{2}, \ldots, i_{n+1} \in \Sigma_{A\left(\mathcal{C}_{1}\right)} .
$$

For the block map $\Phi: B_{n+1}\left(X_{A\left(\mathcal{C}_{1}\right)}\right) \longrightarrow \Sigma_{B\left(\mathcal{C}_{2}\right)}$, let us denote by $\phi:=\Phi_{\infty}^{[0, n]}: X_{A\left(\mathcal{C}_{1}\right)} \longrightarrow$ $X_{B\left(\mathcal{C}_{2}\right)}$ the sliding block code with memory 0 and anticipation $n$ (see [12, p. 15]). The moving block code

$$
\varphi_{\Phi}: X_{A} \longrightarrow X_{B}
$$

is a map defined by

$$
\varphi_{\Phi}:=h_{\mathcal{C}_{2}}^{-1} \circ \phi \circ h_{\mathcal{C}_{1}}
$$

Hence the diagram

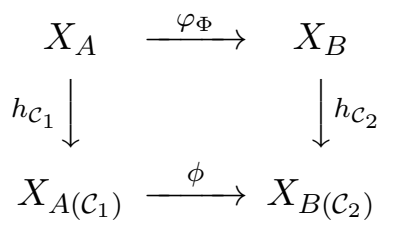

commutes.

If both right Markov codes $\mathcal{C}_{1}, \mathcal{C}_{2}$ are trivial right Markov codes, then the coded block map and the moving block code are block map and sliding block code in the ordinary sense.

Lemma 12. Let $\left(X_{A}, \sigma_{A}\right)$ and $\left(X_{B}, \sigma_{B}\right)$ be one-sided topological Markov shifts. Then they are elementary coded equivalent if and only if there exists a homeomorphism $\varphi_{\Phi}$ : $X_{A} \longrightarrow X_{B}$ of moving block code for some block map $\Phi: B_{n+1}\left(X_{A\left(\mathcal{C}_{1}\right)}\right) \longrightarrow \Sigma_{B\left(\mathcal{C}_{2}\right)}$ with right Markov codes $\mathcal{C}_{1}$ for $\left(X_{A}, \sigma_{A}\right)$ and $\mathcal{C}_{2}$ for $\left(X_{B}, \sigma_{B}\right)$, respectively. 
Proof. Since $\varphi_{\Phi}: X_{A} \longrightarrow X_{B}$ is a homeomorphism if and only if $\phi: X_{A\left(\mathcal{C}_{1}\right)} \longrightarrow X_{B\left(\mathcal{C}_{2}\right)}$ is a homeomorphism. Since $\phi: X_{A\left(\mathcal{C}_{1}\right)} \longrightarrow X_{B\left(\mathcal{C}_{2}\right)}$ is always shift-commuting, we know that $\varphi_{\Phi}: X_{A} \longrightarrow X_{B}$ is a homeomorphism of moving block code if and only if $\phi: X_{A\left(\mathcal{C}_{1}\right)} \longrightarrow$ $X_{B\left(\mathcal{C}_{2}\right)}$ is a topological conjugacy. The latter is equivalent to the condition that $\left(X_{A}, \sigma_{A}\right)$ and $\left(X_{B}, \sigma_{B}\right)$ are elementary coded equivalent. Hence we have the assertion.

In [13], a notion of continuous orbit equivalence of one-sided topological Markov shifts was defined in the following way. Two one-sided topological Markov shifts $\left(X_{A}, \sigma_{A}\right)$ and $\left(X_{B}, \sigma_{B}\right)$ are said to be continuously orbit equivalent if there exist continuous maps $k_{1}, l_{1}$ : $X_{A} \rightarrow \mathbb{Z}_{+}$such that $\sigma_{B}^{k_{1}(x)}\left(h\left(\sigma_{A}(x)\right)\right)=\sigma_{B}^{l_{1}(x)}(h(x))$ for $x \in X_{A}$, and similarly there exist continuous maps $k_{2}, l_{2}: X_{B} \rightarrow \mathbb{Z}_{+}$such that $\sigma_{A}^{k_{2}(y)}\left(h^{-1}\left(\sigma_{B}(y)\right)\right)=\sigma_{A}^{l_{2}(y)}\left(h^{-1}(y)\right)$ for $y \in X_{B}$. The following proposition is a key to show Theorem [14.

Proposition 13. Let $A$ be an irreducible, non permutation matrix with entries in $\{0,1\}$. Let $\mathcal{C}$ be a right Markov code for $\left(X_{A}, \sigma_{A}\right)$. Then the one-sided topological Markov shift $\left(X_{A(\mathcal{C})}, \sigma_{A(\mathcal{C})}\right)$ is continuously orbit equivalent to $\left(X_{A}, \sigma_{A}\right)$.

Proof. Put $B=A(\mathcal{C})$. By Lemma 4 with the unique factorization property of $\mathcal{C}$, for any $x=\left(x_{n}\right)_{n \in \mathbb{N}} \in X_{A}$ there exists a unique increasing sequence $1<k_{1}<k_{2}<\cdots$ of positive integers and $i_{1}, i_{2}, \cdots \in \Sigma_{B}$ such that

$$
x_{\left[1, k_{1}\right)}=\omega\left(i_{1}\right), \quad x_{\left[k_{1}, k_{2}\right)}=\omega\left(i_{2}\right), \quad \ldots \quad x_{\left[k_{n}, k_{n+1}\right)}=\omega\left(i_{n+1}\right), \quad \ldots
$$

That is, $x$ has a unique factorization:

$$
x=\omega\left(i_{1}\right) \omega\left(i_{2}\right) \cdots \omega\left(i_{n}\right) \cdots .
$$

Let $h: X_{A} \longrightarrow X_{B}$ be the standard coding homeomorphism $h_{\mathcal{C}}$ defined by

$$
h(x)=\left(i_{1}, i_{2}, i_{3}, \ldots\right) \quad \text { for } \quad x=\omega\left(i_{1}\right) \omega\left(i_{2}\right) \cdots \omega\left(i_{n}\right) \cdots .
$$

Since

$$
A(\mathcal{C})\left(i_{n}, i_{n+1}\right)=A\left(r\left(\omega\left(i_{n}\right)\right), s\left(\omega\left(i_{n+1}\right)\right)\right)=A\left(x_{k_{n}-1}, x_{k_{n}}\right)=1,
$$

we have $h(x) \in X_{B}$. It is easy to see that $h: X_{A} \longrightarrow X_{B}$ is continuous. As $\mathcal{C}$ has unique factorization property, $h$ is bijective, so that it is a homeomorphism. Let $U_{\omega(i)}$ be the cylinder set of $X_{A}$ beginning with the word $\omega(i)$. As $\mathcal{C}$ is a prefix code with unique factorization property, we have a disjoint union $X_{A}=\sqcup_{i \in \Sigma_{B}} U_{\omega(i)}$ so that we have a disjoint union

$$
X_{A}=\bigsqcup_{\left(i_{1}, i_{2}, \ldots, i_{L}\right) \in B_{L}\left(X_{B}\right)} U_{\omega\left(i_{1}\right) \omega\left(i_{2}\right) \cdots \omega\left(i_{L}\right)}
$$

For any $x \in U_{\omega\left(i_{1}\right) \omega\left(i_{2}\right) \cdots \omega\left(i_{L}\right)} \subset X_{A}$, we set $l_{1}(x)=L, k_{1}(x)=k(i)$, where $k(i)$ is the number $k$ determined by (11). As $\omega\left(i_{1}\right) \omega\left(i_{2}\right) \cdots \omega\left(i_{L}\right)=x_{\left[1, k_{L}\right)}$, its length is $k_{L}-1$. We then have $h(x)=\left(i_{1}, i_{2}, \ldots, i_{L}\right) h\left(x_{\left[k_{L}, \infty\right)}\right)$ so that

$$
\sigma_{B}^{l_{1}(x)}(h(x))=\sigma_{B}^{L}(h(x))=h\left(x_{\left[k_{L}, \infty\right)}\right) .
$$

On the other hand, by (11),

$$
\sigma_{A}(x)=\sigma_{A}\left(\omega\left(i_{1}\right)\right) \omega\left(i_{2}\right) \cdots \omega\left(i_{L}\right) x_{\left[k_{L}, \infty\right)}=\omega\left(j_{1}\right) \omega\left(j_{2}\right) \cdots \omega\left(j_{k}\right) x_{\left[k_{L}, \infty\right)}
$$


so that

$$
h\left(\sigma_{A}(x)\right)=\left(j_{1}, j_{2}, \cdots j_{k}\right) h\left(x_{\left[k_{L}, \infty\right)}\right) .
$$

We then have

$$
\sigma_{B}^{k_{1}(x)}\left(h\left(\sigma_{A}(x)\right)=\sigma_{B}^{k}\left(h\left(\sigma_{A}(x)\right)\right)=h\left(x_{\left[k_{L}, \infty\right)}\right)\right.
$$

and hence

$$
\sigma_{B}^{k_{1}(x)}\left(h\left(\sigma_{A}(x)\right)=\sigma_{B}^{l_{1}(x)}(h(x)) .\right.
$$

We will next study the inverse $h^{-1}: X_{B} \longrightarrow X_{A}$. For $y=\left(i_{1}, i_{2}, i_{3}, \ldots\right) \in X_{B}$ with $i_{1}, i_{2}, i_{3}, \cdots \in \Sigma_{B}$ consider

$$
h^{-1}(y)=\omega\left(i_{1}\right) \omega\left(i_{2}\right) \omega\left(i_{3}\right) \cdots=\left(x_{1}, \ldots, x_{k_{1}-1}, x_{k_{1}}, \ldots, x_{k_{2}-1}, x_{k_{2}}, \ldots\right) .
$$

Put

$$
l_{2}(y)=k_{n}-1\left(=\ell\left(i_{n}\right)\right) \quad \text { for } \quad y \in U_{i_{n}} \subset X_{B}
$$

Since $X_{B}=\sqcup_{i_{j} \in \Sigma_{B}} U_{i_{j}}$, the map $l_{2}: X_{B} \longrightarrow \mathbb{Z}_{+}$is continuous map. We also put $k_{2}(y)=0$ for all $y \in X_{B}$. As $\sigma_{B}(y)=\left(i_{2}, i_{3}, \ldots\right)$, we have

$$
h^{-1}\left(\sigma_{B}(y)\right)=\omega\left(i_{2}\right) \omega\left(i_{3}\right) \cdots=\left(x_{k_{1}}, \ldots, x_{k_{2}-1}, x_{k_{2}}, \ldots\right)
$$

so that

$$
\begin{aligned}
\sigma_{A}^{l_{2}(y)}\left(h^{-1}(y)\right) & =\sigma_{A}^{k_{1}-1}\left(x_{1}, \ldots, x_{k_{1}-1}, x_{k_{1}}, \ldots, x_{k_{2}-1}, x_{k_{2}}, \ldots\right) \\
& =\left(x_{k_{1}}, \ldots, x_{k_{2}-1}, x_{k_{2}}, \ldots\right)
\end{aligned}
$$

and hence we have

$$
\sigma_{A}^{k_{2}(y)}\left(h^{-1}\left(\sigma_{B}(y)\right)\right)=h^{-1}\left(\sigma_{B}(y)\right)=\sigma_{A}^{l_{2}(y)}\left(h^{-1}(y)\right) .
$$

We thus see that $\left(X_{A}, \sigma_{A}\right)$ and $\left(X_{B}, \sigma_{B}\right)$ are continuously orbit equivalent.

The following is a main result of the paper.

Theorem 14. Let $A$ and $B$ be irreducible non permutation matrices. If the one-sided topological Markov shifts $\left(X_{A}, \sigma_{A}\right)$ and $\left(X_{B}, \sigma_{B}\right)$ are coded equivalent, then they are continuously orbit equivalent.

Proof. We first note that continuous orbit equivalence in one-sided topological Markov shifts is an equivalence relation ([13, Theorem 1.1]). We may assume that $\left(X_{A}, \sigma_{A}\right) \underset{\text { code }}{\approx}$ $\left(X_{B}, \sigma_{B}\right)$. Take a right Markov code $\mathcal{C}_{1}\left(\right.$ resp. $\left.\mathcal{C}_{2}\right)$ for $\left(X_{A}, \sigma_{A}\right)\left(\operatorname{resp} .\left(X_{B}, \sigma_{B}\right)\right)$ such that $\left(X_{A\left(\mathcal{C}_{1}\right)}, \sigma_{A\left(\mathcal{C}_{1}\right)}\right)$ is topologically conjugate to $\left(X_{B\left(\mathcal{C}_{2}\right)}, \sigma_{B\left(\mathcal{C}_{2}\right)}\right)$. Since topological conjugacy implies continuous orbit equivalence, we conclude that $\left(X_{A}, \sigma_{A}\right)$ and $\left(X_{B}, \sigma_{B}\right)$ are continuously orbit equivalent, because of Proposition 13

Example 15. 1. Let $A_{1}=\left[\begin{array}{ll}1 & 1 \\ 1 & 0\end{array}\right]$. Define $\mathcal{C}_{1}=\{1,21\}$. Then $\mathcal{C}_{1}$ is a right Markov code. Put $\omega(1)=1, \omega(2)=21$. Since

$$
s(\omega(1))=r(\omega(1))=r(\omega(2))=1, \quad s(\omega(2))=2,
$$


we have $A\left(\mathcal{C}_{1}\right)(i, j)=A(i, j)=1$ for all $i, j=1,2$ so that

$$
A\left(\mathcal{C}_{1}\right)=\left[\begin{array}{ll}
1 & 1 \\
1 & 1
\end{array}\right]=[2]
$$

Therefore $\left(X_{A_{1}}, \sigma_{A_{1}}\right) \underset{\text { code }}{\sim}\left(X_{[2]}, \sigma_{[2]}\right)$ and hence $\left(X_{A_{1}}, \sigma_{A_{1}}\right)$ is continuously orbit equivalent to the full 2-shift. This fact is already seen in [13].

2. Let $A_{2}=\left[\begin{array}{lll}0 & 1 & 0 \\ 0 & 0 & 1 \\ 1 & 1 & 0\end{array}\right]$. Define $\mathcal{C}_{2}=\{12,23,323,312\}$. Then $\mathcal{C}_{2}$ is a right Markov code. Put $\omega(1)=12, \omega(2)=23, \omega(3)=323, \omega(4)=312$. Since

$s(\omega(1))=1, \quad r(\omega(1))=r(\omega(4))=s(\omega(2))=2, \quad r(\omega(2))=r(\omega(3))=s(\omega(3))=s(\omega(4))=3$,

we have

$$
A\left(\mathcal{C}_{2}\right)=\left[\begin{array}{llll}
0 & 0 & 1 & 1 \\
1 & 1 & 0 & 0 \\
1 & 1 & 0 & 0 \\
0 & 0 & 1 & 1
\end{array}\right] .
$$

As the total column amalgamation of the matrix $A\left(\mathcal{C}_{2}\right)$ is the matrix $A\left(\mathcal{C}_{1}\right)$ in (3)). Hence the one-sided topological Markov shift $\left(X_{A\left(\mathcal{C}_{2}\right)}, \sigma_{A\left(\mathcal{C}_{2}\right)}\right)$ is topologically conjugate to the full 2-shift $\left(X_{[2]}, \sigma_{[2]}\right)$. Theherefore $\left(X_{A_{2}}, \sigma_{A_{2}}\right) \underset{\text { code }}{\sim}\left(X_{[2]}, \sigma_{[2]}\right)$.

3. Let $A_{3}=\left[\begin{array}{lll}1 & 1 & 1 \\ 1 & 0 & 1 \\ 1 & 0 & 0\end{array}\right]$. Define $\mathcal{C}_{3}=\{1,21,31,231\}$. Then $\mathcal{C}_{3}$ is a right Markov code.

Put $\omega(1)=1, \omega(2)=21, \omega(3)=31, \omega(4)=231$. Since

$s(\omega(1))=r(\omega(1))=r(\omega(2))=r(\omega(3))=r(\omega(4))=1, \quad s(\omega(2))=s(\omega(4))=2, \quad s(\omega(3))=3$,

we have

$$
A\left(\mathcal{C}_{3}\right)=\left[\begin{array}{llll}
1 & 1 & 1 & 1 \\
1 & 1 & 1 & 1 \\
1 & 1 & 1 & 1 \\
1 & 1 & 1 & 1
\end{array}\right]=[4]
$$

Hence the one-sided topological Markov shift $\left(X_{A\left(\mathcal{C}_{3}\right)}, \sigma_{A\left(\mathcal{C}_{3}\right)}\right)$ is the full 4-shift $\left(X_{[4]}, \sigma_{[4]}\right)$. Theherefore $\left(X_{A_{3}}, \sigma_{A_{3}}\right) \underset{\text { code }}{\sim}\left(X_{[4]}, \sigma_{[4]}\right)$.

Related results to classification of Cuntz-Krieger algebras are seen in [5], [14].

Acknowledgments: The author would like to thank the referee for his/her careful reading the first draft of the paper. This work was supported by JSPS KAKENHI Grant Numbers 15K04896, 19 K03537.

\section{References}

[1] M-P. BÉAL, Codage Symbolique, Masson, Parris 1993. 
[2] J. Berstel And D. Perrin, Theory of codes, Academic Press, London (1985).

[3] F. Blanchard and G. Hansel, Systems codés, Theor. Computer Sci. 44(1986), pp. 17-49.

[4] F. Blanchard and G. Hansel, Sofic constant-to-one extensions of subshifts of finite type, Proc. Amer. Math. Soc. 112(1991), pp. 259-265.

[5] M. Enomoto, M. Fujin And Y. Watatani, $K_{0}$-groups and classifications of CuntzKrieger algebras, Math. Japon. 26(1981), pp. 443-460.

[6] D. Fiebig, Common closing extensions and finitary regular isomorphisms for synchronized systems, in Symbolic Dynamics and Its Applications, Contemporary Mathematics 135(1992), pp. 125-138.

[7] D. FiEBIG, Common extensions and hyperbolic factor maps for coded systems, Ergodic Theory Dynam. Systems 35(1995), pp. 517-534.

[8] D. Fiebig And U. -R. Fiebig, Covers for coded system, in Symbolic Dynamics and Its Applications, Contemporary Mathematics 135(1992), pp. 139-180.

[9] T. Giordano, I. F. Putnam and C. F. Skau, Topological orbit equivalence and $C^{*}$-crossed products, J. Reine Angew. Math. 469(1995), pp. 51-111.

[10] R. H. Herman, I. F. Putnam and C. F. Skau, Ordered Bratteli diagrams, dimension groups and topological dynamics, Internat. J. Math. 3(1992),pp. 827-864.

[11] B. P. Kitchens, Symbolic dynamics, Springer-Verlag, Berlin, Heidelberg and New York (1998).

[12] D. Lind AND B. Marcus, An introduction to symbolic dynamics and coding, Cambridge University Press, Cambridge (1995).

[13] K. Matsumoto, Orbit equivalence of topological Markov shifts and Cuntz-Krieger algebras, Pacific J. Math. 246(2010), 199-225.

[14] K. Matsumoto, Some remarks on orbit equivalence of topological Markov shifts and Cuntz-Krieger algebras, Yokohama Math. J. 58(2012), pp. 41-52.

[15] K. Matsumoto And H. Matui, Continuous orbit equivalence of topological Markov shifts and Cuntz-Krieger algebras, Kyoto J. Math. 54(2014), pp. 863-878.

[16] K. Matsumoto and H. Matui, Full groups of Cuntz-Krieger algebras and HigmanThompson groups, Groups Geom. Dyn. 11(2017), pp. 499-531. 